\title{
A ROBUST CONTROL APPROACH FOR THE FORMATION FLYING OF MULTIPLE SPACECRAFT
}

\author{
M. MESBAHI; F. Y. HADAEGH ${ }^{\dagger}$ \\ Jet Propulsion Laboratory \\ California Institute of Technology \\ 4800 Oak Grove Drive \\ Mail Stop: 198-326 \\ Pasadena, CA 91109-8099 \\ Fax : 818-393-4440
}

Keywords : Formation flying; robust control; disturbance rejection; linear matrix inequality

\begin{abstract}
We present a disturbance rejection mechanism for the formation flying of multiple spacecraft based on a robust control approach in terms of an $H_{\infty}$ control problem. The corresponding $H_{\infty}$ control problem is then solved numerically using linear matrix inequalities.
\end{abstract}

\section{Introduction}

Formation flying (FF) has been identified as an enabling technology for many of the NASA's $21^{\text {st }}$ century missions, among them, the Deep Space 3 and the Terrestrial Planet Finder. Formation flying involves flying a group of spacecraft in a particular pattern while maintaining precise (but often time varying) relative position, velocity, attitude, and angular velocity, with respect to each other [2], [6]. Since traditional spacecraft control is often concerned with measuring and maintaining the same quantities for a single spacecraft with respect to an inertial reference frame, the analogous FF control and estimation problems are often an order of magnitude more challenging than those encountered traditionally for a single spacecraft. In order to make the FF control problems at least similar to the single spacecraft case, an approach based on leaderfollowing has been proposed by Wang and Hadaegh [7] (also refer to [8]). The basic idea in leader-following (LF) is to designate a particular frame (or multiple frames) in the FF as the reference frame(s) and measure and control the states of the rest of the formation with respect to them.

The present paper addresses the problem of designing a control law for the follower spacecraft in an LF formation

\footnotetext{
-Email: mesbahiohafez.jpl.nasa.gov

IEmail: fred.y.hadaeghojpl.nasa.gov
}

which is guaranteed to attenuate the effects of environmental disturbances on the performance of the leader following. The results of the paper are in direct relevance to those reported [4] and [7] for the formation keeping problem. Building on the basic feedback linearization scheme in [7], we propose a control mechanism for the formation in the presence of disturbance forces and torques based on the $H_{\infty}$ methodology. The linear matrix inequality (LMI) [1] formulation of the corresponding $H_{\infty}$ problem is then used to design a candidate controller which is, in the $H_{\infty}$ sense, optimal.

The organization of the paper is as follows. In $\$ 2$ the assumptions which constitute the framework for the formation keeping problem are listed. In $\S 3$ and $\$ 4$ the basic facts and the formulation of the problem considered in the paper are presented, followed by the design techniques which introduce the $H_{\infty}$ formulation of the disturbance rejection. A numerical example and the corresponding simulation result are then presented in $\$ 5$.

First a few words on the notation. Formation flying consists of flying a group of spacecraft in a particular pattern. To be able to express the time evolution of the formation and design the corresponding control laws, it is convenient that a reference frame is attached to each spacecraft. We shall always assume that these reference frames are induced from a dextral set of three orthonormal vectors. Let the formation have $n$ spacecraft labeled as $1,2, \ldots, n$. Let $\mathcal{F}^{i}$ denote the reference frame attached to the $i$-th spacecraft; $\mathcal{F}^{\mathbf{I}}$ on the other hand shall designate the inertial reference frame. For the inertia and the mass of the $i$-th spacecraft we use $I^{i}$ and $m^{\mathbf{i}}$, respectively. The force and torque acting upon $\mathbf{i}$ are denoted by $f^{1}$ and $r^{\mathbf{1}} ;$ for the mass normalized force we used $u^{i}:=\frac{f^{i}}{m^{i}}$. The time derivative with respect to $\mathcal{F}^{-1}$ shall be denoted by $\frac{d}{d t_{1}}$; likewise, $\frac{d}{d t}$ will be used for the time derivative with respect to $\mathcal{F}^{1}$. $r^{j}$ denotes the position of the origin of $\mathcal{F}^{1}$ with respect to $\mathcal{F}^{j} ; r^{i}$ is the position of the origin of $\mathcal{F}^{i}$ with respect to $\mathcal{F}^{\mathbf{l}}$. The desired position of the origin of $\mathcal{F}^{l}$ 


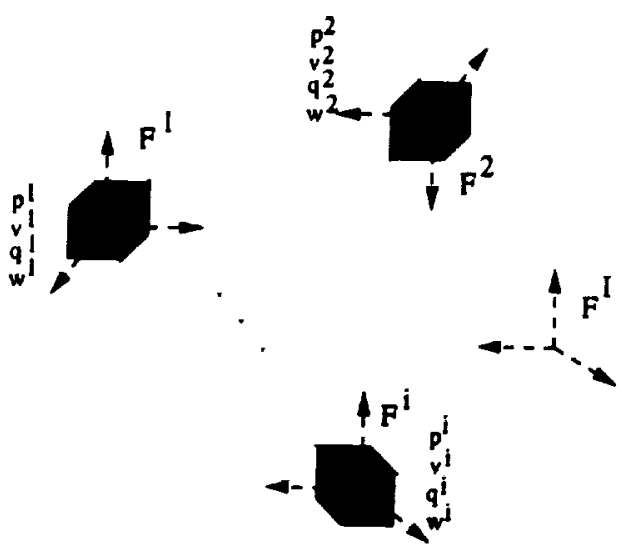

Figure 1: Formation Coordinates

with respect to $\mathcal{F}^{\mathrm{J}}$ shall be denoted by $r_{d}^{\mathrm{J}}$, and by $r_{d}^{\mathbb{J}}$ when $\mathbf{j}=\mathbf{I}$. The velocity of the origin of $\mathcal{F}$ with respect to $\mathcal{F}^{j}$, the velocity of the origin of $\mathcal{F}^{\mathfrak{T}}$ with respect to $\mathcal{F}^{\mathbf{I}}$, the desired velocity of the origin of $\mathcal{F}^{-}$with respect to $\mathcal{F}^{j}$ , and the desired velocity of the origin of $\mathcal{F}^{-1}$ with respect to $\mathcal{F}^{\mathrm{I}}$, shall be denoted by $v^{\mathbf{i j}}, v^{\mathbf{i}}, v_{d}^{\mathbf{j}}$, and $v_{d}^{\mathbf{i}}$, respectively. Similar notation is used for the angular velocity of $\mathcal{F}^{\mathfrak{i}}$ with respect to $\mathcal{F}^{\mathbf{j}}: \omega^{\mathbf{i}}$ is the angular velocity of $\mathcal{F}^{\mathbf{i}}$ with respect to $\mathcal{F}^{\mathbf{j}}$ and $\omega_{d}^{\mathbf{j}}$ is the desired angular velocity of $\mathcal{F}^{\mathrm{i}}$ with respect to $\mathcal{F}^{\mathrm{j}}$ (refer to Figure 1). The cross product matrix induced by the vector $x=\left[\begin{array}{lll}x_{1} & x_{2} & x_{3}\end{array}\right]^{T}$ is the matrix,

$$
[x]:=\left[\begin{array}{ccc}
0 & -x_{3} & x_{2} \\
x_{3} & 0 & -x_{1} \\
-x_{2} & x_{1} & 0
\end{array}\right]
$$

\section{Assumptions}

We consider a group of spacecraft which are to be kept in a particular formation pattern. The attitude control of the spacecraft in particular is not considered in the present study. However, the orientation of the spacecraft does play a role in the formation keeping problem, since the angular velocity of the spacecraft effects the measurements of relative distances and velocities. The following assumptions are explicitly made in the present paper:

1. One spacecraft is designated as the leader in the formation; the rest are referred to as the followers.

2. The leader chooses its control force (to acquire the desired position, etc.) independent of the followers' dynamics.

3. The leader communicates its control action to the followers at each instant of time.

4. The followers can measure, for example via the Autonomous Formation Flying sensor (AFF) [5], relative distance and velocities to the leader.
5. The followers have knowledge of their own absolute positions, velocities, and angular velocity, and their attitude with respect to an inertial frame.

6. A commanded differential vector with respect to the leader, expressed in the inertial frame, is supplied to the followers during a particular finite time interval.

7. Uncertainties, in terms of disturbance forces and torques on the follower spacecraft are present. These disturbances are causes by solar pressure, gravity gradient, aerodynamic, or magnetic forces.

8. Uncertainties, in the spacecraft dynamics model, as well as in the communication channel, are considered to be negligible. We shall present results pertaining to some of these issues in the subsequent papers.

Under these assumptions, the follower chooses its control force and torque based on the knowledge of its own dynamics and the control that was used by the leader to track a desired trajectory. We note that relaxing some of these assumptions result in a significant change in the techniques which can be used to address the formation keeping problem.

\section{First, Feedback Linearization}

Under the stated assumptions in $\$ 2$, we consider the scenario where the leader's position, with respect to $\mathcal{F}^{\mathbf{I}}$, evolves according to,

$$
\frac{d r^{1}}{d t}=v^{1}, \quad \frac{d v^{1}}{d t}=\frac{f^{1}}{m^{1}}=u^{1} .
$$

The control force $f^{1}$ is chosen independent of the followers according to some mission objectives, optimality criteria, etc.

Recall that the first and second derivatives of a vector $A$ in $F^{1}$ and $F^{l}$ are related by the following relation,

$$
\frac{d A}{d t}=\frac{d A}{d t_{i}}+\omega^{i} \times A,
$$

where $\omega^{\mathbf{1}}$ is the angular velocity of $F^{\mathbf{1}}$ with respect to $F^{\mathbf{I}}$. In particular,

$$
\frac{d \omega^{i}}{d t}=\frac{d \omega^{i}}{d t_{i}}+\omega^{i} \times \omega^{i}=\frac{d \omega^{i}}{d t_{i}},
$$

stating that the rate of change of the angular velocity is independent of the frame of reference.

Differentiating both sides of (3.1) with respect to $F^{\mathbf{I}}$ we obtain

$\frac{d^{2} A}{d t^{2}}=\frac{d^{2} A}{d t_{i}^{2}}+\frac{d \omega^{\mathbf{i}}}{d t_{\mathrm{i}}} \times A+2 \omega^{\mathbf{i}} \times \frac{d A}{d t_{\mathrm{i}}}+\omega^{\mathbf{1}} \times\left(\omega^{\mathrm{i}} \times A\right)$.

Let,

$$
r_{d}^{1}(t)=r^{1}(t)+h^{\mathbf{i}}(t), \quad t_{0} \leq t \leq t_{f} ;
$$


the error is thus,

$$
\begin{aligned}
e^{1}(t) & =r_{d}^{1}(t)-r^{\mathbf{i}}(t) \\
& =r^{11}(t)+h^{1}(t),
\end{aligned}
$$

where $r^{11}$ is the vector from (the origin of) $F^{\prime}$ to (the origin of) $F^{1}$, i.e., the position of the leader with respect to the $i$-th follower spacecraft coordinates. We like to obtain an expression which describes the time evolution of $e^{l}$ in $F^{i}$. From (3.3) one has,

$$
\frac{d^{2} e^{\mathrm{I}}(t)}{d t^{2}}=\frac{d^{2}\left(r^{1 \mathrm{i}}\right)(t)}{d t^{2}}+\frac{d^{2} h^{\mathrm{i}}(t)}{d t^{2}}
$$

however,

$$
\begin{aligned}
\frac{d^{2}\left(r^{1}\right)(t)}{d t^{2}} & =\frac{d^{2}\left(r^{1}(t)-r^{1}(t)\right)}{d t^{2}} \\
& =u^{1}(t)-u^{1}(t) .
\end{aligned}
$$

In view of (3.2) we have,

$$
\begin{array}{r}
\frac{d^{2} e^{\mathbf{I}}(t)}{d t_{i}^{2}}+\frac{d \omega^{\mathbf{I}}(t)}{d t_{i}} \times e^{\mathbf{i}}(t)+2 \omega^{\mathbf{i}}(t) \times \frac{d e^{\mathbf{I}}(t)}{d t_{1}} \\
+\omega^{\mathbf{i}}(t) \times\left(\omega^{\mathbf{i}}(t) \times e^{\mathbf{I}}(t)\right) \\
=\left(u^{\mathbf{1}}(t)-u^{\mathbf{i}}(t)\right)+\frac{d^{2} h^{\mathbf{i}}(t)}{d t^{2}},
\end{array}
$$

where $u^{i}$ represents the total normalized force acting on the $i$-th spacecraft, i.e.,

$$
u^{l}(t)=u_{c}^{i}(t)+u_{d}^{i}(t)
$$

The last term on the right hand side of (3.6) can of course be represented in $F^{i}$ as,

$$
\begin{aligned}
\frac{d^{2} h^{\mathbf{i}}(t)}{d t_{\mathbf{i}}^{2}}+\frac{d \omega^{\mathbf{i}}(t)}{d t_{\mathbf{i}}} & \times h^{\mathbf{i}}(t)+2 \omega^{\mathbf{i}}(t) \times \frac{d h^{\mathbf{i}}(t)}{d t_{\mathbf{i}}} \\
& +\omega^{\mathbf{i}}(t) \times\left(\omega^{\mathbf{l}}(t) \times h^{\mathbf{i}}(t)\right) .
\end{aligned}
$$

The rate of change of the angular velocity $\omega^{\mathbf{i}}$ with respect to $F^{\mathbf{i}}$ or $F^{\mathbf{1}}$ is related to the torque applied on the spacecraft via the Euler's equation,

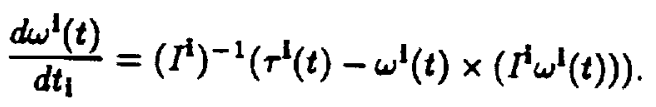

Again the term $r^{1}$ represents the total torque on the $i$-th spacecraft, i.e.,

$$
\tau^{\mathbf{i}}(t)=\tau_{c}^{\mathbf{i}}(t)+\tau_{d}^{\mathbf{i}}(t)
$$

Now, (3.6) represents how the error vector $e^{i}$ evolves in $F^{i}$. We would like to obtain an expression for $u_{c}^{i}$, such that the origin is the globally asymptotically stable limit point of the trajectories defined by (3.6) in the presence of environmental disturbances $u_{d}^{i}$ and $\tau_{d}^{i}$. For this purpose we let.

$$
\begin{aligned}
& x_{1}(t)=e^{i}(t), \\
& x_{2}(t)=\frac{d e^{i}(t)}{d t_{1}}:=\dot{x}_{1}(t), \\
& x_{3}(t)=\omega^{1}(t) .
\end{aligned}
$$

The dynamics of the $i$-th spacecraft can thus be expressed as,

$$
\begin{aligned}
\dot{x}_{1}(t) & =x_{2}(t) \\
\dot{x}_{2}(t) & =-2 x_{3}(t) \times x_{2}(t)-\left(I^{\mathbf{i}}\right)^{-1}\left(\tau_{c}^{\mathbf{i}}(t)+\tau_{d}^{\mathbf{j}}(t)\right. \\
& \left.-x_{3}(t) \times I^{\mathbf{i}} x_{3}(t)\right) \times x_{1}(t)-x_{3}(t) \times\left(x_{3}(t) \times x_{1}(t)\right) \\
& +\left(u^{1}(t)-u_{c}^{\mathbf{i}}(t)-u_{d}^{\mathbf{i}}(t)\right)+\frac{d^{2} h^{\mathbf{i}}(t)}{d t^{2}}, \\
\dot{x}_{3}(t) & =\left(I^{\mathbf{i}}\right)^{-1}\left(\tau_{c}^{\mathbf{i}}(t)+\tau_{d}^{\mathbf{i}}(t)-x_{3}(t) \times I^{\mathbf{i}} x_{3}(t)\right)
\end{aligned}
$$

The differential equations (3.9)-(3.11) describe a nonlinear dynamical system whose state represents the evolution of the position error, position rate error, and the angular velocity of the follower spacecraft, in the follower's coordinate system. In general, one would like to choose the control action such that the error terms go to zero, while certain optimality conditions, and state and control constraints are satisfied. Since designing non-conservative optimal nonlinear controllers in their full generality is a formidable task, one often restores to less ambitious objectives, via for example feedback linearization.

We notice that the parameters available for the control purposes are control force $u_{c}^{1}$ and torque $\tau_{c}^{i}$. However, the control torque might be independently used to obtain a desired orientation during the maneuver, in which case, one would merely focus on obtaining an expression for $u_{c}^{\mathbf{i}}$.

Suppose that the control force and torque are represented as,

$$
\begin{gathered}
u_{c}^{\mathbf{i}}(t)=\bar{u}_{c}^{\mathbf{i}}(t)-u_{f l}^{\mathbf{i}}(t), \\
\tau_{c}^{\mathbf{i}}(t)=\bar{\tau}^{\mathbf{i}}(t)+\tau_{f l}^{\mathbf{i}}(t),
\end{gathered}
$$

where,

$$
\begin{array}{r}
u_{f l}^{\mathbf{i}}(t):=2 x_{3}(t) \times x_{2}(t)+x_{3}(t) \times\left(x_{3}(t) \times x_{1}(t)\right) \\
-u^{\mathbf{1}}(t)-\frac{d^{2} h^{\mathbf{i}}(t)}{d t^{2}}-\left(I^{\mathbf{i}}\right)^{-1} \bar{\tau}_{c}^{\mathbf{i}}(t) \times x_{1}(t), \\
\tau_{f_{1}}^{\mathbf{j}}(t)=x_{3}(t) \times I^{\mathbf{i}} x_{3}(t) .
\end{array}
$$

The subscript ' $f$ ' above is used to denote the 'feedback linearization' term. The dynamics is thus simplified to,

$$
\begin{aligned}
& \dot{x}_{1}(t)=x_{2}(t), \\
& \dot{x}_{2}(t)=-\bar{u}_{c}^{\mathbf{i}}(t)-u_{d}^{\mathbf{i}}(t)-\left(I^{\mathbf{i}}\right)^{-1} \tau_{d}^{\mathbf{i}}(t) \times x_{1}(t), \\
& \dot{x}_{3}(t)=\left(I^{\mathbf{1}}\right)^{-1}\left(\dot{r}_{c}^{\mathbf{i}}(t)+\tau_{d}^{\mathbf{i}}(t)\right) .
\end{aligned}
$$
Let $T=-\left(I^{\mathbf{i}}\right)^{-1}\left[\tau_{d}^{\mathbf{i}}\right]$. From the statistics of $\bar{\tau}_{d}^{\mathbf{i}}$, we con-
struct the set

$$
\Omega \subseteq \Re^{3 \times 3} \text { such that } T \in \Omega
$$

with a probability which can be chosen to be arbitrary close to one; now consider the convex hull of $\Omega$. For the purpose of the present discussion we shall assume that the convex hull is a polytope in $\Re^{3 \times 3}$. Thus, there exists matrices $T_{1}, \ldots, T_{1}$, such that

$$
T \in \operatorname{Co}\left\{T_{1}, \ldots, T_{l}\right\}
$$


where Co denotes the operation of taking the convex hull of a set.

The dynamics of the leader following can thus be represented as,

$$
\begin{gathered}
{\left[\begin{array}{l}
\dot{x}_{1}(t) \\
\dot{x}_{2}(t) \\
\dot{x}_{3}(t)
\end{array}\right]=\left[\begin{array}{lll}
0 & I & 0 \\
T & 0 & 0 \\
0 & 0 & 0
\end{array}\right]\left[\begin{array}{l}
x_{1}(t) \\
x_{2}(t) \\
x_{3}(t)
\end{array}\right]} \\
+\left[\begin{array}{cc}
0 & 0 \\
-I & -\left(I^{1}\right)^{-1} \\
0 & \left(I^{1}\right)^{-1}
\end{array}\right]\left[\begin{array}{l}
\bar{u}_{c}^{1}(t) \\
\bar{\tau}_{c}^{1}(t)
\end{array}\right] \\
+\left[\begin{array}{cc}
0 & 0 \\
-I & -\left(I^{1}\right)^{-1} \\
0 & \left(I^{1}\right)^{-1}
\end{array}\right]\left[\begin{array}{l}
u_{f}^{\mathrm{j}}(t) \\
\tau_{d}^{d}(t)
\end{array}\right]
\end{gathered}
$$

Denote by,

$$
\begin{aligned}
& A_{i}:=\left[\begin{array}{ccc}
0 & I & 0 \\
T_{i} & 0 & 0 \\
0 & 0 & 0
\end{array}\right], \quad i=1, \ldots, l, \\
& B_{u}=\left[\begin{array}{cc}
0 & 0 \\
-I & -\left(I^{1}\right)^{-1} \\
0 & \left(I^{1}\right)^{-1}
\end{array}\right], \quad B_{w}:=\left[\begin{array}{cc}
0 & 0 \\
-I & -\left(I^{1}\right)^{-1} \\
0 & \left(I^{1}\right)^{-1}
\end{array}\right],
\end{aligned}
$$

and let,

$$
u(t):=\left[\begin{array}{c}
\bar{u}_{c}^{\mathbf{i}}(t) \\
\bar{\tau}_{c}^{\mathbf{i}}(t)
\end{array}\right], \quad \text { and } \quad w(t):=\left[\begin{array}{l}
u_{d}^{\mathbf{i}}(t) \\
\tau_{d}^{\mathbf{f}}(t)
\end{array}\right],
$$

The dynamical equations which describe the evolution of the follower spacecraft can therefore be summarized as the following linear differential inclusion [1], [3],

$$
\dot{x}(t)=A x(t)+B_{u} u+B_{w} w(t)
$$

where,

$$
A \in \mathrm{Co}\left\{A_{1}, \ldots, A_{l}\right\} \text {. }
$$

The output equation can generically be represented as,

$$
z(t)=C_{z} x(t)+D_{z u} u(t)+D_{z w} w(t) .
$$

In the subsequent section, we shall build on the linearization and the embedding procedure described above to propose a state feedback linear controller for the formation keeping problem which attenuates the effects of the disturbance vector $\left[\begin{array}{l}u_{d}^{i} \\ \tau_{d}^{d}\end{array}\right]$ on the state of the follower spacecraft.

\section{RMS Gain and State Feedback Synthesis}

We model the disturbance vector $w(t):=\left[\begin{array}{l}u_{d}^{1}(t) \\ \tau_{d}^{1}(t)\end{array}\right]$, as a stationary stochastic process having a finite RMS norm, defined to be,

$$
\|w(t)\|_{\text {RMS }}:=\left(\lim _{T \rightarrow \infty} \frac{1}{T} \int_{0}^{T} w(t)^{\prime} w(t) d t\right)^{1 / 2} .
$$

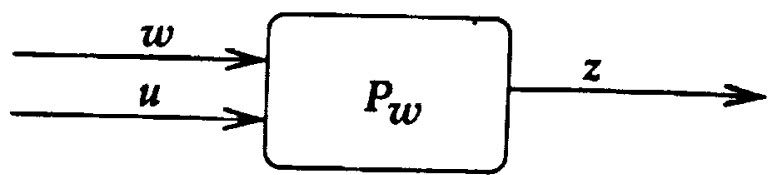

Figure 2: The open loop block diagram for the follower spacecraft (the plant $P$ represents one of the $G_{i}$ 's).

It is known that for an ergodic wide-sense stationary stochastic signal, the RMS norm can be expressed in terms of the power spectral density function $S(\omega)$,

$$
\|w(t)\|_{\mathrm{RMS}}=\text { Trace } \frac{1}{2 \pi} \int_{-\infty}^{\infty} S_{w}(\omega) d w .
$$

Now consider the follower spacecraft dynamics after the feedback linearization, as represented by (3.13) (Figure 2). For each $T_{i}$ one as a linear time invariant system; let the corresponding transfer matrix be denoted by $G_{i}(s)$. The RMS gain of a transfer matrix $G_{i}$ or its $H_{\infty}$ norm is the largest ratio of the RMS norm of the noise signal $w$ to the RMS norm of the output signal $z$, i.e.,

$$
\left\|G_{i}(s)\right\|_{\infty}:=\sup _{\|w(t)\|_{\mathrm{RMS}} \neq 0} \frac{\|z(t)\|_{\mathrm{RMS}}}{\|w(t)\|_{\mathrm{RMS}}} .
$$

It can also be shown that,

$$
\left\|G_{i}(s)\right\|_{\infty}:=\sup _{\omega} \sigma_{\max }\left(G_{i}(j \omega)\right)
$$

where $\sigma_{\max }\left(G_{i}(j \omega)\right)$ denotes the maximum singular value of the (complex) matrix $G_{i}(j \omega)[1]$.

We now focus on proposing a state feedback control law which has as its goal, the minimization of the RMS gain of the resulting family of closed loop feedback systems which represent the follower spacecraft dynamics.

\subsection{State Feedback Synthesis}

In this section we present a state feedback control which aims to minimize the RMS gain of the family of closed loop systems which represent the dynamics of follower spacecraft. The follower dynamics with the controller in the feedback loop can thus be represented as in Figure 2 , where $K$ is considered to be a constant state feedback gain.

For simplicity of the present discussion, we shall assume that $D_{z w}=0$, i.e., that the noise does not directly affect the output signal $z$. Let $u(t)=K x(t)$ in $(3.14)-(3.15)$; thus,

$$
\begin{aligned}
\dot{x}(t) & =\left(A+B_{u} K\right) x(t)+B_{w} w(t), \\
A & \in \operatorname{Co}\left\{A_{1}, \ldots, A_{l}\right\}, \\
z(t) & =\left(C_{z}+D_{z u} K\right) x(t) .
\end{aligned}
$$

In order to minimized the RMS gain from $w$ to $z$, consider the Lyapunov function $V(x, t)=x(t)^{\prime} P x(t)$, where 


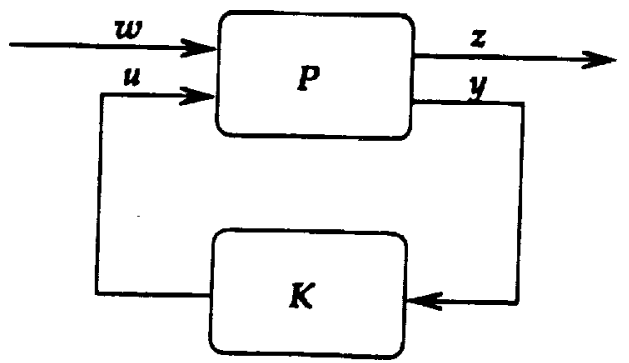

Figure 3: The block diagram for the follower spacecraft with the state feedback controller (the plant $P$ represents one of the $G_{i}$ 's).

the matrix $P$ is positive definite. Suppose that $P$ is chosen such that there exists a nonnegative number $\gamma$ satisfying [1],

$$
\frac{d}{d t} V(x, t)+z(t)^{\prime} z(t)-\gamma^{2} w(t)^{\prime} w(t) \leq 0 .
$$

Thereby,

$$
\begin{array}{r}
\lim _{T \rightarrow \infty} \frac{1}{T} \int_{0}^{T} z(t)^{\prime} z(t) d t \\
-\lim _{T \rightarrow \infty} \frac{1}{T} \int_{0}^{T} \gamma^{2} w(t)^{\prime} w(t) d t \leq 0,
\end{array}
$$

since $V(x(T)) \geq 0$. Thus one can deduce that,

$$
\frac{\|z(t)\|_{\text {RMS }}}{\|w(t)\|_{\text {RMS }}} \leq \gamma,
$$

i.e., one can bound the RMS gain of the closed loop system by $\lambda$ by an appropriate selection $K$ which admits a quadratic Lyapunov function with the desired properties.

Expanding the condition (4.16) for the family of transfer matrices $G_{i}$ 's $(i=1, \ldots, l)$, one obtains,

$$
\begin{array}{r}
\left(A x(t)+B_{u} u(t)+B_{w} w(t)\right)^{\prime} P_{x}(t) \\
+x(t)^{\prime} P\left(A x(t)+B_{u} u(t)+B_{w} w(t)\right) \\
-\left(C_{x} x(t)+D_{z u} u(t)+D_{z w} w(t)\right)^{\prime}\left(C_{z} x(t)\right. \\
\left.+D_{z u} u(t)+D_{z w} w(t)\right)-\gamma^{2} w(t)^{\prime} w(t) \leq 0,
\end{array}
$$

for all $A \in \operatorname{Co}\left\{A_{1}, \ldots A_{1}\right\}$ and all $x \in \Re^{9}$

After some simplifications, and setting $Q:=P^{-1}$, and $Y=K Q(4.17)$ can be written as,

$$
\begin{gathered}
{\left[\begin{array}{cc}
X_{1 i} & X_{2 i} \\
X_{2 i}^{\prime} & X_{3 i}
\end{array}\right] \leq 0} \\
\quad(i=1, \ldots, l),
\end{gathered}
$$

where,

$$
\begin{array}{r}
X_{1 i}=A_{i} Q+Q A_{i}^{\prime}+B_{u} Y+Y^{\prime} B_{u}{ }^{\prime}+B_{w} B_{w}{ }^{\prime} \\
X_{2 i}=\left(C_{z} Q+D_{3 u} Y\right)^{\prime} \\
X_{3 i}=-\gamma^{2} I .
\end{array}
$$

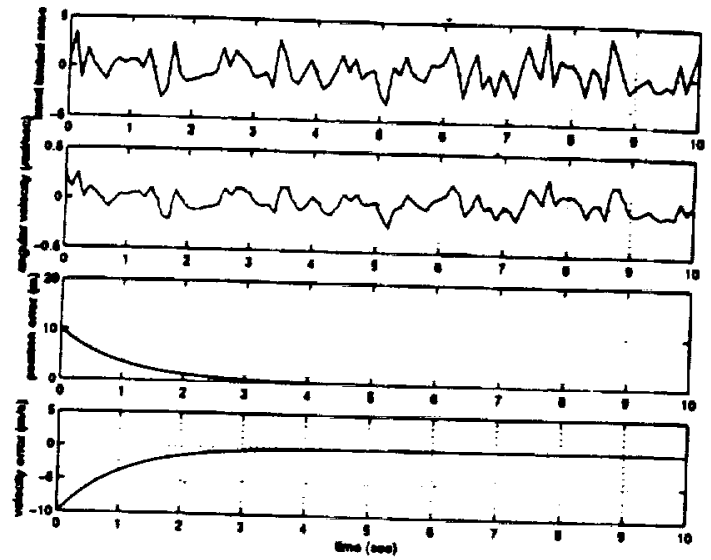

Figure 4: Simulation results for a representative scenario

Thus, in order to find a controller which aims to minimize the RMS gain of the family of closed loop systems representing the follower spacecraft dynamics, we are led to solve the following semi-definite program,

$$
\begin{gathered}
\min _{\gamma, Y, Q \gamma} \\
Q>0 \\
{\left[\begin{array}{cc}
A_{i} Q+Q A_{i}^{\prime}+B_{u} Y+Y^{\prime} B_{u}{ }^{\prime}+B_{w} B_{w}{ }^{\prime} & \left(C_{z} Q+D_{z u} Y\right)^{\prime} \\
\left(C_{z} Q+D_{z u}\right) Y & -\gamma^{2} I
\end{array}\right]} \\
(i=1, \ldots, l)
\end{gathered}
$$

and then let $K=Y Q^{-1}$.

\section{An Example}

In this section we provide an example and the corresponding simulation result for the proposed state feedback synthesis procedure discussed above. For this purpose, given the matrices $A_{1}, \ldots, A_{l}, B_{u}$, and $B_{w}$, as in (3.14), we chose the following matrices for the simulation purposes,

$$
C_{z}=I \text { and } D_{z u}=0 .
$$

The LMItool, an optimization package developed by $\mathrm{EI}$ Ghaoui, Nikoukhah, and Delebercque based on the SP code of Boyd and Vandenberghe, implementing the pri$\mathrm{mal} / \mathrm{dual}$ interior point method for solving semi-definite programs, was used to solve the SDP (4.18)-(4.18). The disturbance force and torque was modeled as a band limited white noise. The simulation result is depicted in Figure 3.

\section{Conclusion}

We proposed a disturbance rejection mechanism for the formation keeping problem. The disturbance rejection problem is first formulated in terms of a family of $H_{\infty}$ optimization problems. We then proceeded to solve these 
$H_{\infty}$ problem using their LMI formulations via the recently proposed interior point methods. A numerical example was provided to demonstrate the usefulness of the proposed approach for formation flying in the presence of RMS bounded disturbance forces and torques.

\section{Acknowledgments}

The research of the authors was carried out at the Jet Propulsion Laboratory, California Institute of Technology, under a contract with the National Aeronautic and Space Administration.

\section{References}

[1] S. P. Boyd, L. EL Ghaoui, E. Feron, and V. Balakrishnan. Linear Matrix Inequalities in System and Control Theory. SIAM, Philadelphia, (1994).

[2] A. B. Decou. Multiple spacecraft optical interferometry- Preliminary feasibility assessment. Technical report, Jet Propulsion Laboratory, (1991).

[3] A. F. Filippov. Differential Equations with Discontinuous Righthand Sides. Kluwer Academic Publishers, The Netherlands, (1988).

[4] F. Y. Hadaegh, W. M. Lu, and P. K. C. Wang. Adaptive control of formation flying spacecraft for interferometry. In Proceedings of IFAC/IFORS/IMACS/IFIP Symposium on Large Scale Systems: Theory and Applications, (1998).

[5] G. Purcell, D. Kuang, S. Lichten S-C. Wu, and L. Young. Autonomous formation flyer (AFF) sensor technology development. In 21st Annual AAS Guidance and Control Conference, February 4-8, (1998).

[6] R. Stachnik, K. Ashlin, and K. Hamilton. SpaceStation-SAMSI: A spacecraft array for Michelson spatial interferometry. Bulletin of the American Astronomical Society, 16(3):818-827, (1984).

[7] P. K. C. Wang and F. Y. Hadaegh. Coordination and control of multiple microspacecraft moving in formation. Journal of the Astronautical Sciences, 44(3):315355 , (1996).

[8] P. K. C. Wang, F. Y. Hadaegh, and K. Lau. Synchronized formation rotation and attitude control of multiple free-flying spacecraft. Journal of Guidance, Control and Dynamics, 21(6), (1998). 